In Press, Clinical Psychological Science, Special Issue on COVID-19 Pandemic and Mental Health

A Comparison of Mental Health Outcomes in the United States and Italy at Different Levels of Cumulative COVID-19 Prevalence

\author{
Anthony D. Mancini \\ Pace University \\ Gabriele Prati \\ University of Bologna
}

Correspondence should be addressed to: Anthony D. Mancini, Department of Psychology, Marks Hall, Pace University, Pleasantville, NY 10570. Email: amancini@pace.edu. 


\begin{abstract}
How does the prevalence of COVID-19 impact people's mental health? In a preregistered study $(N=857)$, we sought to answer this question by comparing demographically matched samples in four regions in the United States and Italy with different levels of cumulative COVID-19 prevalence. No main effect of prevalence emerged. Rather, prevalence region had opposite effects, depending on the country. New York City participants (high prevalence) reported more general distress, PTSD symptoms, and COVID-19 worry than San Francisco (low prevalence). Conversely, Campania participants (low prevalence) reported more general distress, PTSD symptoms, and COVID-19 worry than Lombardy (high prevalence). Consistent with these patterns, COVID-19 worry was more strongly linked with general distress and PTSD symptoms in New York than San Francisco, whereas COVID-19 worry was more strongly linked with PTSD in Campania than Lombardy. In exploratory analyses, media exposure predicted and mapped on to geographic variation in mental health outcomes.
\end{abstract}

Keywords: COVID-19, mental health, sociocultural factors, media exposure, posttraumatic stress 


\section{A Comparison of Mental Health Outcomes in the United States and Italy at Different Levels of Cumulative COVID-19 Prevalence}

New York City and the Lombardy region of Italy were early epicenters of the COVID-19 pandemic, drawing international attention for a steep rise in cases and deaths in the first months of the pandemic in 2020 (McKinley, 2020; Winfield, 2020). In response, stringent lockdowns were imposed in Italy and parts of the United States in March, 2020. In combination with the threat of harm from the virus, these lockdown measures raised substantial concerns about the mental health impact of the pandemic, and early media reports suggested that these concerns were well-founded (Brooks, 2020). Although the mental health effects of the early lockdowns appear to have been relatively small and highly variable (Prati \& Mancini, 2021), there are sound reasons to think that lockdowns and worries about COVID-19 infection have distinctive effects on mental health, such as loneliness, on the one hand, and anxiety symptoms, on the other. However, given that lockdowns often co-occurred with high COVID-19 case intensity, it is difficult to isolate their unique effects. One strategy is to conduct a natural experiment by comparing regions that implemented similar lockdowns despite having substantially different COVID-19 case rates. In the current preregistered study, we used this strategy to examine the mental health effects of an objective marker of COVID-19 risk and long-term persistence, cumulative COVID-19 case and death prevalence. We were particularly interested in the impact of this objective marker on mental health, whether it was attenuated by trust in institutional authorities, whether it was explained by COVID-19 worries, and whether its effects showed variation across two national contexts, the United States and Italy.

\section{COVID-19 Prevalence and Mental Health}


Fear of pathogens is believed to be an evolved adaptation (Hart, 1990), and widely varying research suggests that pandemics and other infectious disease crises can result in mental health consequences (Huremović, 2019). Both the H1N1 pandemic (also known as swine flu) and the Ebola crisis were associated with negative mental health effects (Huremović, 2019; Jalloh et al., 2018; Thompson et al., 2017). More recently, the COVID-19 pandemic has been linked to negative mental health consequences in both population-based studies (Holman et al., 2020) and in meta-analyses (Prati \& Mancini, 2021). A plausible explanation for these effects involves worries about the pandemic and COVID-19 infection. That is, the greater the likelihood of infection in a region, the more worried people in that region would be. If that is the case, regional variation in COVID-19 prevalence should be associated with mental health outcomes (Mancini, 2020). In China, for example, anxiety was higher in regions with more COVID-19 cases (Zhao et al., 2020). In the United States, fear related to COVID-19 was also higher in regions with greater COVID-19 infection rates (Fitzpatrick et al., 2020). Thus, prior evidence suggests that worries about infection pose risks for mental health and these effects may vary according to disease prevalence.

In addition to these risks, the wide-ranging and stringent lockdown policies implemented to control virus spread could have negative effects on mental health. However, if lockdown policies are implemented at the same time that case prevalence increases, it is not possible to distinguish the effects of lockdowns versus the effects of high prevalence. One way of disentangling these effects is to compare regions with similar lockdown policies but different degrees of cumulative COVID-19 prevalence. Such a comparison can test whether the prevalence of COVID-19 is associated with mental health outcomes independent of lockdown policies. A further value of comparing regions with different levels of cumulative prevalence is 
that it can help to ascertain mental health effects of lockdowns. If lockdowns are the predominant influence on mental health, it might be assumed that variations in COVID-19 prevalence would contribute little beyond the effects of lockdowns. However, if differences do emerge in regions with different levels of COVID-19 prevalence, it would at minimum provide evidence that any effect of lockdowns is not uniform across environments that differ in COVID-19 prevalence.

A number of factors could mitigate the mental health effects of COVID-19 prevalence, but one with particular relevance is trust in institutional authorities. Institutional trust contributes generally to well-being (Hudson, 2006) and has been associated with reduced mental health consequences of COVID-19 (e.g., Lee, 2020; Mana \& Sagy, 2020; Prati, 2021). Trust in institutions should be particularly important under conditions of high stress (Mishler \& Rose, 2001), specifically with regard to increased COVID-19 prevalence, given the critical role of governmental policy in addressing rising caseloads. Some studies have suggested that governmental trust tended to increase during the pandemic (Kye \& Hwang, 2020; Sibley et al., 2020), but these increases also depended on perceived institutional performance (Kye \& Hwang, 2020). Although these findings suggest a protective effect of institutional trust, a countervailing concern is that institutional trust is also correlated with media consumption (Strömbäck et al., 2016; Yang et al., 2014), a known risk factor for negative mental health consequences of mass adversity (Garfin et al., 2020), and with political identification (Thomson Jr. et al., 2019), a potential operational confound. These considerations suggest that institutional trust should mitigate the mental health consequences of high COVID-19 prevalence but also that media exposure and political identification might confound this relationship.

\section{Country-Level Comparisons}


Policy responses to the pandemic varied considerably across countries and broader regions of the world. Moreover, there are important cultural dimensions of psychological functioning that could also play a role in mental health outcomes (Hofstede, 1980). A crossnational comparison can illuminate whether these differences contribute to variation in the mental health impact of the pandemic. One widely-studied characteristic that shows substantial cultural variation is individualism, the tendency to construe the self as largely independent of the broader group (Hofstede, 1980; Triandis, 1995). The United States and other English-speaking countries generally report the highest levels of individualism (Hofstede, 2019). By contrast, Italy has lower levels of individualism than the United States (Burton et al., 2021; Hofstede, 2019). Although more individualistic countries generally have higher levels of depression and anxiety symptoms (Kessler \& Üstün, 2008), we did not formulate a priori hypotheses regarding countrylevel differences in mental health outcomes. Instead, we sought to examine whether country moderated any observed effects or whether findings replicated across the two countries.

\section{Operationalizing COVID-19 Cumulative Prevalence}

Early reports of the pandemic in the West centered on the Lombardy region of Italy and New York City in the United States. Both areas saw rapidly accumulating cases and very high case fatality rates in the early months of the pandemic. In New York City, 223,737 cases and 18,889 deaths were recorded due to COVID-19 related causes from early March to the end of July (Johns Hopkins University Center for Systems Science and Engineering, 2021), a case fatality rate of $8.4 \%$. Similarly, in the Lombardy region of Italy from late February to July, there were 96,219 cases and 16,806 deaths, an even higher case fatality rate of $17.5 \%$ (Johns Hopkins University Center for Systems Science and Engineering, 2021). By contrast, other regions of Italy and the United States in this period had remarkably low cumulative cases and deaths from 
COVID-19, notably the city of San Francisco and the Campania region of Italy. In contrast to New York City, San Francisco reported just 7,177 cases and only 67 deaths during the same period, a case fatality rate of $0.9 \%$. Similarly, the Campania region in Italy reported 4,999 cases and 435 deaths, a case fatality rate of $8.7 \%$.

\section{Current Study}

Do these regional differences in cumulative COVID-19 prevalence have consequences for people's mental health? In a 2 x 2 preregistered study (https://osf.io/p7sve), we hypothesized they would. In two national contexts (United States and Italy), we examined four regions that differed substantially in COVID-19 cumulative prevalence but implemented broadly equivalent lockdown measures. Participants were assessed from late July to early September 2020 from regions with high and low COVID-19 cases and deaths (high: New York and Lombardy; low: San Francisco and Campania). To address selection threats and enhance generalizability, the high-prevalence region was sampled to reflect census-matched demographic characteristics (age, gender, education, income, and race/ethnicity) and a matched sample was selected from the lowprevalence region. We focused on symptoms of general distress, specific worries about COVID19, and posttraumatic stress disorder symptoms related to COVID-19 as mental health outcomes. We hypothesized that participants in regions with high COVID-19 prevalence would show more general distress, posttraumatic stress disorder (PTSD) symptoms, and COVID-19 worry, compared to regions with lower prevalence. Furthermore, we hypothesized that this effect would be weaker for participants with higher institutional trust. We also examined whether the effect of COVID-19 prevalence region on general distress and PTSD symptoms would be accounted for by COVID-19 worry. ${ }^{1}$ Finally, in preregistered exploratory analyses, we examined whether these 
effects differed in two countries with distinct national cultures, levels of individualism, and policy responses to the pandemic, the United States and Italy.

\section{Method}

\section{Participants and Procedure}

Participants were recruited by survey firms in the United States (US) and Italy. On the basis of a preregistered power analysis, we sought a total sample of 800 participants (400 in each country; 200 in each region), which would afford $>80 \%$ power to detect small to medium-sized effects $(f=.12)$ for a 4-group design. In the United States, we recruited participants from an optin Qualtrics internet panel drawn from various sources, including consumer websites, member referrals, targeted email lists, social media, and customer loyalty programs. Qualtrics collects demographic data upon enrollment (age, race/ethnicity, education, gender, income, and zip code), allowing us to select a sample on the basis of census-matched New York City demographics and residence in a zip code in one of the five boroughs of New York City. Only participants who met criteria were screened into the survey by Qualtrics. A matched demographic sample was selected in the County of San Francisco based on zip code residence. The median date of data collection by region was as follows: New York City = August 9th, 2021; San Francisco = August 19th, 2021; Lombardy = July 31st, 2021; Campania = July 31st, 2021. See Figure 1 for a depiction of the timing of the assessments in relation to cumulative deaths over time. Data and materials for this study can be found here: https://osf.io/h6cmw/

During the time the survey was available (July 29th to September 10th), five hundred and fifty-eight participants screened into and completed surveys; 143 participants were removed by Qualtrics on the basis of unreliable survey completion times (25.6\%), defined as completion durations less than $1 / 2$ of the median of a preliminary batch of surveys ( $n=34 ; M d=10.23$ 
minutes). Thus, participants who completed the survey in approximately less than 5 minutes were removed from our final sample as part of data-blind quality control procedures implemented by Qualtrics. This left a sample of 415 complete surveys ( $n=210$, New York; $n=$ 205, San Francisco). All surveys were confidential and self-administered. Our final sample in New York City closely approximated 2019 Census data (United States Census Bureau, 2019). Specifically, participants were $50.7 \%$ female (US Census $=52.3 \%$ ), $40 \%$ white (US Census $=$ 42.7\%), 23.3\% black (US Census $=24.3 \%), 19 \%$ Latino/Hispanic (US Census $=29.1 \%), 14.3 \%$ Asian $($ US Census $=14.1 \%), 2.9 \%$ multiracial $($ US Census $=3.6 \%)$, and reported a median income of $\$ 75,000-99,000$ (US Census $=\$ 63,998$ ) and being at least college educated for 49.1\% (US Census $=38.1 \%$ ). Our final sample in San Francisco, compared to New York City residents, showed no differences in gender, age, income, education $\left(p_{\mathrm{s}}>.57\right)$, the proportion of white $(p=.50)$, Latino $(p=.42)$, or multiracial participants $(p=.15)$. There were more Black participants in New York and more Asian participants in San Francisco $\left(p_{s}<.001\right)$. See Supplemental Table S1 for detailed demographic comparisons of regions in the United States and Italy. This data collection was approved by the Pace University Institutional Review Board (\#1592051-5).

In Italy, similar procedures were used to recruit and assess participants in Lombardy and Campania. For the present study we used the profiled online panel of Demetra Sas, an online survey company that collects and analyzes data for academic research, polls, and market research. Demetra has a profiled Italian online panel whose age, gender, and region distribution could be compared to census data. This Italian online panel was collected mainly offline through invitations to the panel, made following CATI (Computer-Assisted Telephone Interviewing) or CAMI (Computer Assisted Mobile Interviewing) surveys. The survey company Demetra 
recruited participants on the basis of postal codes in the regions of Lombardy and Campania. During the time the survey was available in Italy (July $31^{\text {st }}$ to September $2^{\text {nd }}$ ), 511 participants screened into and completed surveys; 56 participants were removed by Demetra on the basis of unreliable survey completion times (11.0\%), leaving a sample of 455 complete surveys $(n=231$; $n=224)$. Participants from the Lombardy region were selected to reflect population-level demographics and a matched sample was recruited in the Campania region. Specifically, our final sample in Lombardy approximated 2019 population-level demographics in Italy (ISTAT, 2021). Specifically, participants were $51.1 \%$ female (ISTAT $=51.0 \%$ ), 3.9\% non-native (ISTAT $=11.5 \%)$ and reported a mean age of $45.55($ ISTAT $=45.0)$, and $58.9 \%$ were at least college educated (ISTAT $=62.9 \%$ ). Our final sample in Campania showed no differences in gender, age, education, or immigration status from the Lombardy sample $\left(p_{\mathrm{s}}>.68\right)$ but did differ in income $(p$ $=.008$ ). This data collection was approved by the Bioethics Committee of Università di Bologna.

To compare regions on COVID-19 cases and deaths, we used the cumulative totals reported on the median date that participants completed assessments within each region (Johns Hopkins Center for Science and Engineering, 2021). To adjust for population differences, we standardized these figures per 100,000 population based on 2019 population Census data for New York City and San Francisco (Census Bureau, 2019) and 2020 ISTAT data for Lombardy and Campania (ISTAT, 2021). The total number of cases in New York City was 226,042 (2,640 per 100,000 population) compared to San Francisco's 8,683 cases (983 per 100,000 population). The total number of deaths in New York City was 18,944 (221 per 100,000 population) compared to San Francisco's 83 deaths (9 per 100,000 population). For Italy, the total number of cases in Lombardy was 96,219 (956 per 100,000 population) compared to Campania's 4,999 (95 per 100,000 population). The total number of deaths in Lombardy was 16,806 (167 per 100,000 
population) compared to Campania's 435 (8 per 100,000 population). See Figure 1 for a visual depiction of the fielding period for assessments in relation to cumulative death totals in the United States and Italy.

These comparisons support substantial differences in cumulative case prevalence and death rates across high and low regions in the United States and Italy. Nevertheless, our surveys were completed at a time when cases and deaths were declining across all regions. In the previous seven days before the median assessment date in each region, New York City reported an average of 239 cases and five deaths per day; San Francisco reported an average of 68 cases and less than one death per day; Lombardy reported an average of 64 cases and less than one death per day; and Campania reported an average of 17 cases and less than one death per day.

\section{Measures}

General distress. In both samples, general distress was assessed using the Depression Anxiety Stress Scales (DASS)-21 (Bottesi et al., 2015; Lovibond \& Lovibond, 1995). The DASS-21 is a self-report measure developed with the specific aim of assessing affective syndromes of depression, anxiety, and tension/stress. Participants were asked to report how many of each of the symptoms depicted in the 21 items applied to them over the past two weeks using a four-point rating ranging from "did not apply to me at all" to "applied to me very much, or most of the time." For the Italian sample, we used an independently validated translation of the scale (Bottesi et al., 2015). We used a sum score in analyses. The higher the score, the more serious the general distress was. Cronbach's alpha coefficients were excellent for both the US and Italian samples $(\alpha=.98$ and .96 respectively).

Posttraumatic stress disorder symptoms (PTSD). PTSD symptoms were assessed using the 8-item Harvard Trauma Questionnaire (Hansen et al., 2010). The PTSD-8 is a short 
screening instrument and had good psychometric properties in three independent samples of disaster victims, whiplash patients, and rape victims (Hansen et al., 2010). Given that the PTSD8 is derived from a selection of the items of the Harvard Trauma Questionnaire, the corresponding eight items of the Italian version of the Harvard Trauma Questionnaire were used for the Italian sample (Caneo, 2018). Participants were asked to rate how often they have experienced symptoms in relation to their experiences during the COVID-19 pandemic in the last month. Questions were phrased specifically to refer to the pandemic (e.g., "How often have you avoided thoughts or feelings associated with the COVID-19 pandemic.") Responses on these items were measured on a 4-point scale ranging from 1 (not at all) to 4 (all the time). The instrument was conceptualized as unidimensional, and the average score provides an estimate of PTSD symptom severity. Internal consistencies were high for both the US and Italian samples $(\alpha$ $=.91$ and .88 respectively).

COVID-19 worry. COVID-19 worry was assessed using a six-item scale derived from previous research on the pandemic influenza H1N1 2009 (Prati, Pietrantoni, \& Zani, 2011a, 2011b). The wording of the items were as follows: (1) "Do you think you are at risk of contracting coronavirus?"; (2) "How often do you worry about contracting coronavirus?”; (3) "How often do you worry about the future in relation to the Coronavirus pandemic?"; (4) "How often do you worry about finances in relation to the Coronavirus pandemic?"; (5) "How often do you worry about school disruptions related to the Coronavirus pandemic?" (6) "How well would you be able to cope if you contracted coronavirus?" Participants were asked to rate the degree to which item applied to them over the past two weeks using a 7 -point scale $(1=$ not at all, $7=$ extremely). Based on parallel analysis, one factor was chosen. Exploratory factor analysis revealed that all items reported acceptable factor loadings (i.e., >.40) with the exception of the 
sixth item ("How well would you be able to cope if you contracted coronavirus?"). Therefore, we decided to exclude this item and used a five-item scale to measure COVID-19 worry. ${ }^{2}$ We averaged responses to derive a mean-item score. Higher scores represent greater worry about the epidemic of COVID-19. Cronbach's alpha for both the US and Italian samples was .82 and .71, respectively.

Institutional trust. Participant's trust in institutions regarding the COVID-19 pandemic was measured using a 4-item scale based on a prior study (Prati, 2021). Participants were asked two questions about their belief and trust in local and state (regional) authorities to handle the challenges of the pandemic: "Do you think the [local authorities or state government] are competent in dealing with the COVID-19 pandemic?" and "Do you trust [local authorities or state government] to keep you safe during the pandemic?" Participants were asked to rate each item on a 7 -point scale $(1=$ not at all, $7=$ extremely $)$. Parallel analysis supported one factor and exploratory factor analysis indicated that all items had acceptable factor loadings (i.e., >.40). We averaged responses to derive a mean-item score. Higher scores represent greater institutional trust in the regional government's capacity to address COVID-19. Cronbach's alpha was satisfactory for both the US and Italian samples ( $\alpha=.94$ and .82 respectively).

Individualism. Participant's individualism was measured using the 4-item subscale of horizontal individualism from the Horizontal \& Vertical Individualism \& Collectivism II scale (Triandis \& Gelfand, 1998). Horizontal individualism assesses the extent to which people strive to be self-reliant and to distinguish themselves from others (e.g., "I'd rather depend on myself than others"). Cronbach's alpha was satisfactory for both the US and Italian samples ( $\alpha$ $=.94$ and .82 respectively). 
Media exposure. Participant's consumption of media related to the COVID-19 pandemic was measured using a 3-item measure. Consistent with recommended practice (de Vreese \& Neijens, 2016), we asked participants to rate different types of media consumption and in relation to a particular topic (i.e., the coronavirus pandemic). Specifically, participants used a 7-point scale (where $1=$ not at all, $4=31-60$ minutes, and $7=3$ hours or more) to indicate how much time they spent per day in the last week: a) reading news articles; b) watching news shows; or c) watching online videos (e.g., YouTube) that relate specifically to the coronavirus pandemic. Such estimates have been found to accurately reflect relative levels of exposure (LaCour \& Vavreck, 2014). Parallel analysis indicated one factor and factor loadings from exploratory factor analysis were acceptable (e.g., > .40). We averaged responses to derive a mean-item score. Higher scores represent greater media exposure. Cronbach's alpha was satisfactory for both the US and Italian samples ( $\alpha=.78$ in both countries).

Political identification. Participant's political identification were measured using a oneitem measure that was rated on a 7 -point scale in the United States, where $1=$ very liberal, $4=$ moderate, and $7=$ very conservative and on a 6-point scale in Italy, where $1=$ left, $3=$ center, and $5=$ right and $6=$ no political orientation. To accommodate the different anchors in the two countries, we stretched the 5-point anchors in Italy to suit a 7-point scale (1 to $1 ; 2$ to $2.5 ; 3$ to 4 ; 4 to $5.5 ; 5$ to 7$)$ and recoded 6 in Italy to the midpoint of the United States scale $(4=$ moderate/center).

Demographic variables. Age, gender, education, income, race/ethnicity, and immigration status were assessed. Age was measured by asking participants to report their age in Italy and to select one of seven categories in the United States $(18-25 ; 26-34 ; 35-44 ; 45-$ 54; $55-64 ; 65-74 ; 75$ and older). To facilitate comparability across the US and Italy samples, 
we derived both a continuous measure of age for the US by taking the midpoint of each category; and a categorical measure of age for Italy by placing continuous ages into the US categories for the US. Education was assessed by asking participants to report their highest level of education, with six response options ranging from middle school or less to Doctoral, MD or law degree (PhD, MD, JD). Income was assessed by asking participants to report which of 9 categories accurately characterized their income in either dollars or euros (from $0-24,000$ to over 200,000). To facilitate relative comparisons of income across each country in our analyses, we standardized the income variable within country and used the $\mathrm{z}$ scored variable in multivariate analyses.

\section{Covariates}

To control for potential confounders, we followed our preregistered plan for including covariates when there were marginal differences $(p<.10)$ in age, gender, ethnicity, education, and income across prevalence regions in each country. We also preregistered the inclusion of a covariate for lockdown duration (the number of days each participant was under some form of regional lockdown restriction). However, in Italy, all restrictions were lifted nationwide on June $15^{\text {th }}$, well before the first assessment. As a result, lockdown duration showed no variation in Italy. In the US, San Francisco instituted a lockdown on March 17 $7^{\text {th }}, 2020$; New York on March $22^{\text {nd }}, 2020$. Some restrictions remained in both cities through the end of September, and there were significant differences between New York and San Francisco in days of lockdown $\left(M_{\text {New }}\right.$

York $=139.8, S D$ New York $=6.79 ; M_{\text {San Francisco }}=153.65, S D$ San Francisco $\left.=9.46\right)$, with participants in San Francisco being exposed to a greater lockdown duration than New York participants $(t=$ 17.05, $p<.001$ ), because of a longer assessment period in San Francisco. Thus, we did not include lockdown duration as a covariate in our combined country analyses. However, we 
retained it for analyses restricted to the United States sample. Finally, we also examined media exposure and political ideology as exploratory covariates in robustness checks.

\section{Statistical Analysis}

Our preregistered analysis plan can be found here: https://osf.io/p7sve. We used SPSS 26.0 for data analysis. We screened data for accuracy, missing data, outliers, and normal distribution assumptions. Outliers were defined using the median absolute deviation $>3$ as the cutoff (Leys, Delacre, Mora, Lakens, \& Ley, 2019). To examine differences across low and highrisk regions within each country, we used independent samples t-tests for continuous variables and non-parametric Mann-Whitney U tests for ordinal variables.

To test hypotheses regarding the effect of cumulative COVID-19 prevalence and institutional trust, we used regression techniques and interaction terms. To test exploratory interactions of region and country, we used General Linear Models (GLM), which provide regression analysis and analysis of variance for one dependent variable by one or more factors and covariates. All continuous variables were grand mean-centered for interaction terms. In the case of violation of normal distribution assumptions, we used bootstrapping as an alternative to parametric estimates for deriving robust estimates of standard errors and confidence intervals (Wright, London, \& Field, 2011). We obtained 95\% percentile confidence intervals using 5000 bootstrapped replications. Either semi-partial squared $r\left(s r^{2}\right)$ or partial eta squared $\left(\eta^{2}\right)$ was reported as estimates of effect size in our multivariate analyses. Following the guidelines of Cohen (1988), values of partial $\eta^{2}$ of $.01, .06$, and .14 were considered small, medium, or large effects. Per our preregistered plan, demographic covariates were included if marginal differences emerged across prevalence regions within country $(p<.10)$. 
A second set of hypotheses proposed that COVID-19 worry would mediate the effect of high prevalence region on general distress and PTSD symptoms. However, as a transparent change, we reformulated this analysis into a multiple regression framework because of the risk of bias in cross-sectional mediation (O'Laughlin et al., 2018). Thus, we examined whether COVID19 worry reduced the unique variance explained by prevalence region in order to assess whether such worry helps to explain the effects of prevalence region. We report the preregistered mediational tests in Supplemental Table S2.

\section{Results}

\section{Descriptive Analyses}

Table 1 shows percentages, means, and standard deviations of demographic and study variables. Scores on COVID-19 worry, PTSD, and general distress ranged from 1 to 7 ( $M=4.36$, $S D=1.29), 1$ to $4(M=2.06, S D=0.74)$, and 0 to $63(M=18.84, S D=14.901)$, respectively. Bivariate correlations $\left(r_{\mathrm{s}}\right)$ revealed that COVID-19 worry correlated positively with PTSD $\left(r_{s}=\right.$ $.51, p<.001)$ and general distress $\left(r_{s}=.37, p<.001\right)$. Also, general distress was positively related to PTSD $\left(r_{s}=.56, p<.001\right)$. (See Supplemental Table S3 for the full correlation matrix by country.) Scores on study variables deviated from the normal distribution. Specifically, moderate skew was found in the scores of PTSD and general distress, as is often the case in clinical measures. Thus, we employed bootstrapping approaches to address this in our regression analyses. Scores on COVID-19 worry, PTSD, and general distress were higher in New York City (high COVID-19 prevalence) compared to San Francisco (low COVID-19 prevalence). In contrast, a reverse pattern was found for Italy, where scores on COVID-19 worry, PTSD, and general distress were lower in Lombardy (high COVID-19 prevalence) compared to Campania (low COVID-19 prevalence). 
Country-level comparisons revealed that United States participants reported more PTSD symptoms ( $p<.001, d=.26)$, institutional trust $(p=.02, d=.16)$, media exposure $(p<.001, d=$ .16 ), and liberal political identification ( $p<.001, d=.25$ ). Consistent with the proposed cultural distinction, participants in the United States reported slightly more individualism ( $p=.03, d=$ $.15)$ than did participants in Italy. ${ }^{3}$ There were no differences in general distress $(p=.41)$ or COVID-19 worry $(p=.15)$ between the United States and Italy. In regional comparisons across countries, a number of differences emerged. New York participants, when compared to Lombardy participants, reported substantially higher levels of PTSD symptoms $(p<.001, d=$ .61 ) and more COVID-19 worry $(p<.001, d=.36)$, but not more general distress $(p=.08)$. New Yorkers also reported considerably more institutional trust $(p<.001, d=.47)$ and media exposure $(p<.001, d=.42)$ than Lombardi. When comparing San Francisco and Campania, participants in San Francisco reported lower levels of PTSD symptoms $(p=.002, d=.30)$ and higher levels of media exposure ( $p=.05, d=.19$ ). However, no differences emerged between San Francisco and Lombardy in PTSD symptoms, COVID-19 worry, institutional trust or individualism $\left(p_{s}>.09\right)$. A final descriptive analysis compared United States and Italian participants to norms for elevated scores on the DASS-21 (Lovibond \& Lovibond, 1995b). These comparisons indicated that mean levels of general distress in all regions were elevated. In comparison to norms, all regions were close to or above normative thresholds for either "mild" or "moderate" elevations in general distress (mild $=16.5$ to 19.5 ; moderate $=19.5$ to 29.5 ). See Supplemental Table S4 for additional statistical information on country-level comparisons.

\section{Does Cumulative COVID-19 Prevalence Predict Worse Mental Health?}

Our primary hypothesis was that high COVID-19 prevalence region would predict increased general distress, PTSD symptoms, and COVID-19 worry. Following our preregistered 
analysis plan, we conducted hierarchical multiple regression analyses for each outcome, entering COVID-19 prevalence region on a first step and a control variable (income) on a second step. These analyses failed to yield support for our hypotheses $\left(p_{\mathrm{s}}>.18\right)$, indicating prevalence region in the combined country sample was unrelated to general distress, PTSD symptoms, and COVID-19 worry. These analyses, however, did not take into account potential differences across the two countries. Thus, we next included country as a moderator of the effect of prevalence region in a set of preregistered exploratory analyses.

\section{Country-level Interactions with Region}

To examine country-level interactions with COVID-19 prevalence region, we used GLMS entering dummy-coded predictors for Country (Italy $=1, \mathrm{US}=0$ ) and for prevalence region (high $=1$, low $=0$ ), a control variable for income, and specified Country $\times$ Region interactions predicting general distress, PTSD symptoms, and COVID-19 worry. See Table 2 for the results of these analyses. For each outcome, we found evidence of a significant interaction of Country $x$ Region $\left(p_{\mathrm{s}}<.006\right)$, suggesting crossover interaction effects in which the effect of prevalence region was opposite depending on the country (see Table 1).

Because the interaction terms were significant in the three GLMs, we did not interpret the direction of the main effects. Interaction plots (profile plots) were used for comparing the estimated marginal means of our three dependent variables (adjusted for covariate) at each level of the factors (see Figure 2). To decompose these patterns, we conducted simple slopes analyses by country, controlling for preregistered covariates of income and lockdown duration (only in the United States analyses, as lockdown duration did not vary in Italy). These analyses revealed that the effect of high prevalence region was opposite across the two countries. In the US, consistent with our hypotheses, high COVID-19 prevalence region positively predicted general distress 
$F(1,414)=20.80, p<.001$, PTSD symptoms $F(1,414)=20.93, p<.001$, and COVID-19 worry $F(1,414)=8.59, p=.004$. By contrast, in Italy, low COVID-19 prevalence region positively predicted general distress $F(1,454)=4.68, p=.03$, PTSD symptoms $F(1,454)=13.52, p<$ .001 , and COVID-19 worry $F(1,454)=6 . .46, p=.01$.

Next we examined exploratory controls for political identification and media exposure. These analyses revealed that political identification had no effect on the interactions of region and country on mental health outcomes. Media exposure also did not alter the interactions of region and country on general distress and PTSD, but it did render the prediction of COVID-19 worry non-significant $(p=.14)$. This suggested that media exposure explained variation in COVID-19 worry across region and country (see Supplemental Table 5 for details of these exploratory analyses).

\section{Does Institutional Trust Reduce the Effect of High COVID-19 Prevalence on Mental}

\section{Health?}

A second hypothesis was that institutional trust would moderate the impact of prevalence region on general distress, PTSD symptoms, and COVID-19 worry. To examine this hypothesis, we conducted multiple regression analyses using our preregistered analysis plan. We entered a dummy-coded predictor for prevalence region (high $=1$, low $=0$ ), institutional trust (grand meancentered), and specified Institutional Trust $\times$ Region interactions predicting general distress, PTSD symptoms, and COVID-19 worry. The results revealed significant interaction effects of region and institutional trust on general distress, $F(1,864)=4.74, p=.03$, and PTSD symptoms, $F(1,864)=6.87, p=.009$, but not COVID-19 worry $F(1,864)=2.32, p=.13$.

Surprisingly, these effects were opposite to our preregistered hypotheses. Instead of attenuating the relationship between high prevalence region and mental health outcomes, 
institutional trust amplified mental health outcomes in the high prevalence but not low prevalence regions. We next examined three-way interactions for Institutional Trust $\times$ Region $\times$ Country. These analyses yielded no three-way effects for general distress, $F(1,863)=.78, p=$ .37 , PTSD symptoms, $F(1,863)=.84, p=.54$, or COVID-19 worry, $F(1,863)=.55, p=.46$. Thus, country did not moderate the observed two-way effects of prevalence region and institutional trust on the outcome variables.

Because the amplifying effect of institutional trust on worse mental health outcomes was counterintuitive, we investigated it further in exploratory robustness checks. We found that media exposure was positively correlated with institutional trust $(r=.21, p<.001)$ and with general distress $(r=.35, p<.001)$, PTSD symptoms $(r=.44, p<.001)$, and COVID-19 worry $(r$ $=.35, p<.001)$. Given the positive direction of these relationships, media exposure could serve as a confound, and thus without controlling it, the estimation of the effect could be artificially inflated (Mehio-Sibai et al., 2005). To address this possibility, media exposure was included as a control to adjust for its effects in a subsequent analysis. With media exposure controlled, both of the original interaction effects were rendered non-significant $\left(p_{\mathrm{s}}>.17\right)$. Although we did not preregister media exposure as a covariate, these findings indicate the unadjusted interaction effects of Region $\times$ Institutional Trust are likely spurious, and as a result, we did not interpret them. ${ }^{4}$ (See Supplemental Figure S1 for a depiction of the interaction effects without adjustment for media exposure. Supplemental Table S6 provides the full regression results.)

\section{Does COVID-19 Worry Explain the Effect of Prevalence Region?}

The next set of analyses examined whether COVID-19 worry accounted for the effects of COVID-19 prevalence region on mental health. These analyses used a hierarchical regression framework to examine the explanatory capacity of COVID-19 worry. Given the cross-over 
interactions previously observed, we first included all lower and higher order country-level interactions. Specifically, we entered Region, Country, COVID-19 worry, and a control variable (income) on a first step, all two-way interaction terms on a second step, and a three-way Region $\times$ Country $\times$ COVID-19 worry interaction term on a final step. These analyses revealed significant three-way interactions for both PTSD, $F(1,862)=8.49, p=.004$, and for general distress, $F(1,862)=8.79, p=.003$.

Next, to better understand these interactions, hierarchical regression analyses were conducted separately for the United States and Italy samples (see Supplemental Table S7 for the full hierarchical regression results by country). In the analyses for the United States, we entered prevalence region and control variables (income and lockdown duration) on a first step and COVID-19 worry on a second step. Prevalence region was a positive predictor for both general distress $\left(B=9.76, S E=2.14,95 \%\right.$ CI $\left.[5.53,13.96], p<.001, s r^{2}=.048\right)$ and PTSD $(B=.47, S E$ $\left.=.10,95 \% \mathrm{CI}[.27, .68], p<.001, s r^{2}=.047\right)$, but when COVID-19 worry was entered on a second step, the unique variance accounted for by prevalence region was reduced by $50 \%$ for general distress $\left(s r^{2}=.024\right)$ and by $54 \%$ for PTSD symptoms (to $s r=.023$ ). In the analyses for Italy, we entered prevalence region and a control variable (income) on a first step and COVID-19 worry on a second step. Consistent with the cross-over interactions previously observed, prevalence region was a negative predictor of both general distress $(B=-2.56, S E=1.18,95 \%$ CI $\left.[-4.48,-.24] p=.03 s r^{2}=.01\right)$ and PTSD $\left(B=-.22, S E=.06,95 \%\right.$ CI $[-.34,-.10], p<.001, s r^{2}$ $=.029)$. The entry of COVID-19 worry on a second step accounted for all of the predictive capacity of prevalence region on general distress, so that it was no longer significant $(B=-1.84$, $S E=1.16,95 \%$ CI $\left.[-4.16, .44], p=.11 s r^{2}=.004\right)$ and $63 \%$ of the predictive capacity for PTSD symptoms (to $s r^{2}=.012$ ). Together these analyses indicated that COVID-19 worry was 
responsible for a substantial proportion of the predictive variance of prevalence region on mental health outcomes.

\section{Prevalence Region Interactions with COVID-19 Worry}

We further decomposed these effects by exploring whether they varied by region. Using the same regression equations, we examined two-way interactions of Region x COVID-19 Worry on an additional step in each country, controlling for income and lockdown duration. In the United States, we observed a significant two-way interaction of Region $\times$ COVID-19 worry on general distress, $F(1,409)=7.42, p=.007$, and on PTSD symptoms, $F(1,409)=4.32, p=$ .04. As shown in the top panel of Figure 2, a simple slopes analysis showed that the relationship between COVID-19 worry and general distress was stronger in the high prevalence region $(B=$ $6.12, S E=.79,95 \% \mathrm{CI}[4.56,7.67], p<.001)$ than the low prevalence region $(B=3.62, S E=$ $.64,95 \%$ CI $[2.34,4.09], p<.001)$. As with general distress, the relationship between COVID19 worry and PTSD was stronger in the high prevalence region $(B=.33, S E=.03,95 \%$ CI $[.26$, $.40], p<.001)$ than the low prevalence region $(B=.24, S E=.03,95 \%$ CI $[.18, .30], p<.001$.

In Italy, this effect showed the opposite pattern as the United States. There was an interaction of Region $\times$ COVID-19 worry, $F(1,450)=4.42, p=.036$, but as shown in the bottom panel of Figure 2, the relationship between COVID-19 worry and PTSD symptoms was stronger in the low prevalence region $(B=.30, S E=.04,95 \% \mathrm{CI}[.26, .41] p<.001)$ than in the high prevalence region $(B=.22, S E=.03,95 \% \mathrm{CI}[.16, .29], p<.001)$. The analysis of general distress did not yield a significant two-way interaction effect $(p=.16)$. Thus, COVID-19 worry was more strongly linked to PTSD symptoms in the low prevalence region of Italy than the high prevalence region. A final set of analyses explored controls for media exposure and political ideology. We found no evidence that their inclusion in the regression analyses altered the 
interaction findings (see Supplemental Table 7), though media exposure did explain additional unique variance in each mental health outcome, over and above COVID-19 worry, prevalence region, and their interaction.

\section{Discussion}

This study compared participants from regions in the United States and Italy that differed substantially in cumulative COVID-19 prevalence but that were similar in lockdown restrictions. We hypothesized that higher cumulative prevalence would be associated with worse mental health outcomes and that institutional trust would be protective against higher prevalence, attenuating its effects on mental health. We did not confirm these hypotheses. Instead, exploratory findings revealed unexpected interactions of prevalence region and country. Across outcomes and analyses, we found that the high prevalence region in the US was associated with worse mental health outcomes, whereas the high prevalence region in Italy was associated with better mental health outcomes. For example, participants in New York City reported higher levels of general distress, PTSD symptoms, and COVID-19 related worry when compared to participants in San Francisco, the low prevalence region. By contrast, Italian participants in Campania, the low prevalence region, reported higher levels of general distress, PTSD symptoms, and COVID-19 worry than participants in the high prevalence Lombardy region. Together, these findings suggested a complex picture in which the relative prior intensity of the pandemic (COVID-19 cumulative prevalence) had divergent mental health associations depending on the national context in which they occurred.

One potential explanation for the relatively better mental health outcomes in Lombardy is altered risk perceptions of COVID-19. One study found that participants living in the less affected regions of Italy were more likely to report fear and to overestimate the perception of the 
outbreak spread compared to people living in more affected regions (Motta Zanin, Gentile, Parisi, \& Spasiano, 2020). Similar findings have been observed in relation to the typhoon eye effect, in which people closest to the epicenter of a disaster experience fewer mental health symptoms than those in outlying regions (Li et al., 2010; Xie et al, 2011). Other evidence suggests that actual infection rates contribute modestly to COVID-19 distress and behaviors, whereas perceived infections are a much stronger predictor of COVID-19 distress and behaviors (Schmidt et al., 2021). Consistent with this possibility, we found higher COVID-19 worry in Campania than Lombardy, suggesting greater vigilance to the potential harms of the pandemic despite substantially lower cumulative prevalence. Because such concerns should be linked to disease prevalence, we expected New York participants to report more worry about COVID-19, and New Yorkers were more worried about COVID-19 than San Franciscans in our study.

We also observed, relative to norms for the DASS-21, that participants in both countries reported elevated distress (Lovibond \& Lovibond, 1995b). New Yorkers' level of general distress was mildly to moderately elevated and San Franciscans were just below the threshold for mild elevations (Lovibond \& Lovibond, 1995b). Similar results were obtained in Italy, with participants in both Lombardy and Campania exceeding norms. One factor that contributed to elevated general distress and PTSD symptoms was COVID-19 worry, an association that was amplified by region. In the US, COVID-19 worry was more strongly linked to general distress and PTSD symptoms in New York City than in San Francisco. Notably, the opposite pattern was observed in Italy, where COVID-19 worry was more strongly linked with PTSD symptoms in Campania than in Lombardy. These findings clearly suggest that worries about COVID-19 contribute variance to worse mental health, but they also show that such worries are not simply attributable to cumulative disease prevalence, an objective risk indicator. Instead, it appears that 
perceptions of the pandemic likely play a key role (Cisler \& Koster, 2010; Kalisch et al., 2015; Mancini, Aldrich, et al., 2020).

This raises the question of what would influence those perceptions. We found no evidence that institutional trust was associated with better mental health outcomes, either as a main effect or as a moderator. Nor did we find evidence that political identification was clearly associated with mental health. By contrast, one factor that emerged unexpectedly was media exposure. In exploratory analyses, we found that COVID-19-related media consumption mapped directly on to the patterns of distress across the two countries, being higher in New York and Campania, and lower in San Francisco and Lombardy. When media exposure was controlled, it also eliminated the interaction of region and country in predicting COVID-19 worry, suggesting media exposure helped to explain variation in worries about COVID-19 across region and country. Media exposure was also a key confounding variable. For example, without controlling for media exposure, institutional trust amplified the effect of prevalence region on mental health outcomes, a result directly opposite to our preregistered prediction. When controls for media exposure were included, the evidence failed to support any interaction effects, suggesting that media exposure's simultaneous positive relationship with institutional trust and mental health accounted for the interaction. Finally, in multivariate analyses, media exposure emerged as a strong predictor (medium to large effect sizes) of general distress, PTSD symptoms, and COVID-19 worry, over and above political identification, country, and prevalence region. These findings are consistent with a growing literature on the deleterious effects of media exposure during mass stressors (Garfin et al., 2020; Hopwood \& Schutte, 2017; Thompson et al., 2017). Nevertheless, our cross-sectional design cannot rule out other unobserved confounds, nor can it 
determine whether COVID-19 related media exposure leads to more distress or distress leads to more consumption of COVID-19 related media.

What explains the effect of prevalence region? When COVID-19 worry was treated as a predictor variable, it explained a large proportion of the effects of prevalence region on general distress and PTSD symptoms. In the United States, COVID-19 worry accounted for over 50\% of the predictive capacity of the high prevalence region on general distress and PTSD symptoms. In Italy, the effect was reversed: COVID-19 worry accounted for a similar proportion of the variance of the low prevalence region on PTSD, and in the case of general distress, it explained the effect of prevalence region entirely. Consistent with the prior results, media exposure also accounted for additional variance in prevalence region, over and above COVID-19 worry. These findings suggest that prevalence region's relationship to mental health outcomes is to a large degree explained by worries about the pandemic and media exposure related to it.

A final set of exploratory analyses examined differences between the United States and Italy, both as a main effect and within prevalence type (e.g., Lombardy vs. New York). We found that the United States participants reported higher levels of COVID-19 worry and more PTSD symptoms than Italian participants. One possible explanation for these country-level differences involves the way lockdown measures were relaxed in the two countries. In both New York City and San Francisco, the most stringent initial shelter-in-place orders were rescinded in early June 2020, but many other lockdown measures remained in place through the end of September. However, in Italy, the initial reopening in May 2020 was followed by a complete reopening on June $15^{\text {th }}, 2020$. Thus, it is possible that the relaxation of lockdown restrictions contributed to better outcomes in Italy. Indeed, comparisons of high prevalence regions across the two countries indicated that Lombardy participants reported better mental health than new 
York participants. We found substantially more PTSD symptoms and COVID-19 worry (as well as media exposure) among New Yorkers than Lombardi. Although these differences are provocative, suggesting that New York experienced more mental health consequences from the pandemic than Lombardy, the samples differed on many variables that could not be controlled across countries, including locality, income, education, and race and ethnicity, as well as unobserved variables, and thus selection threats remain active alternative interpretations of these differences. We would also note that these descriptive findings cannot be generalized to the United States and Italy more broadly, and whether similar findings would be obtained in nationally representative samples cannot be determined from these data.

\section{Limitations and Future Research Directions}

There are a number of methodological limitations of the current investigation. First, we collected data through a self-administered opt-in online questionnaire and, therefore, people without internet service, computers or smartphones were not included. Similarly, there are likely selection biases in such opt-in surveys that we were unable to control for. Although the sample largely reflected census demographics, the extent to which the sample is representative of unobserved characteristics of the population is unknown because we did not use a probabilitybased methodology. We went to considerable lengths to control selection threats, but we cannot rule out that the observed effects are a consequence of factors unrelated to pandemic intensity. Second, our surveys were administered at a time when caseloads were declining across all regions and indeed worldwide. Thus, our operational definition only applied to cumulative COVID-19 burden and not to dynamic changes in the spread of the SARS-cov-2 virus. Third, the fielding period for the assessments differed across the two countries, with Italy completing their assessments, on average, about two weeks earlier than United States participants. These 
differences could introduce confounds. Fourth, cross-sectional survey methods cannot establish causality or directionality among a set of variables. Thus, we cannot rule out that unobserved confounds are responsible for the observed relationships or establish their directionality. Future research using longitudinal or experimental designs can investigate the direction and causal link between COVID-19 worry and mental health outcomes. Fifth, there was some variation in the interactions of COVID-19 worry and prevalence region on mental health, indicating that these effects were more consistent in the United States than Italy. A final limitation is that our sample was not exposed to a criterion A stressor for PTSD (American Psychiatric Association, 2011), limiting the relevance of these findings to research on COVID-19 and PTSD diagnoses.

\section{Conclusions}

Using data from four regions and two countries, this study demonstrates the complexity of COVID-19's effects on mental health. The present findings indicate that cumulative prevalence has associations with mental health that differ substantially according to regional and national factors. Together, these findings underscore the ways psychological phenomena are embedded in situational and cultural contexts, each of which can refract the forces that bear on healthy functioning in the face of the threat of the COVID-19 pandemic. 


\begin{abstract}
Author Contributions
A.D.M. developed the study concept. Both authors contributed to the study design. Data

collection was performed by A.D.M. and G.P. Both authors performed the data analysis and interpretation. A.D.M. drafted the Introduction and Discussion, and G.P. drafted the Method and Results. Both authors provided critical revisions and approved the final version of the paper for submission.
\end{abstract}




\section{References}

Bottesi, G., Ghisi, M., Altoè, G., Conforti, E., Melli, G., \& Sica, C. (2015). The Italian version of the Depression Anxiety Stress Scales-21: Factor structure and psychometric properties on community and clinical samples. Comprehensive Psychiatry, 60, 170-181.

doi:10.1016/j.comppsych.2015.04.005

Brooks, D. (2020). 'I Feel Like I'm Finally Cracking and I Don't Even Know Why'. Retrieved April 29th from https://www.nytimes.com/2020/04/09/opinion/mental-health-isolationcoronavirus.html

Burton, L., Delvecchio, E., Germani, A., \& Mazzeschi, C. (2021). Individualism/collectivism and personality in Italian and American Groups. Current Psychology, 40(1), 29-34. https://doi.org/10.1007/s12144-019-00584-4

Caneo, N. (2018). Adattamento e standardizzazione del test Harvard Trauma Questionnaire (HTQ) in italiano, tramite un campione di tipo non clinico (adaptation and standardization of the Harvard Trauma Questionnaire (HTQ) test in italian, using a nonclinical sample). (Bachelor thesis), Università di Parma. Dipartimento di Medicina e Chirurgia,

Cisler, J. M., \& Koster, E. H. W. (2010). Mechanisms of attentional biases towards threat in anxiety disorders: An integrative review. Clinical Psychology Review, 30, 203-216. doi:10.1016/j.cpr.2009.11.003

Cohen, J. (1988). Statistical Power Analysis for the Behavioral Sciences (second ed.). Hillsdale, NJ: Erlbaum. 
Cucchiarini, V., Caravona, L., Macchi, L., Perlino, F. L., \& Viale, R. (2021). Behavioral changes after the COVID-19 lockdown in Italy. Frontiers in Psychology, 12, 617315-617315. doi:10.3389/fpsyg.2021.617315

de Vreese, C. H., \& Neijens, P. (2016). Measuring Media Exposure in a Changing Communications Environment. Communication Methods and Measures, 10(2-3), 69-80. https://doi.org/10.1080/19312458.2016.1150441

Johns Hopkins Center for Science and Engineering. (2021). Coronavirus (COVID-19) Cases and Deaths Data. Retrieved from https://data.humdata.org/dataset/novel-coronavirus-2019ncov-cases.

Fitzpatrick, K. M., Harris, C., \& Drawve, G. (2020). Fear of COVID-19 and the mental health consequences in America. Psychological Trauma: Theory, Research, Practice, and Policy, 12, S17-S21. doi:10.1037/tra0000924

Garfin, D. R., Silver, R. C., \& Holman, E. A. (2020). The novel coronavirus (COVID-2019) outbreak: Amplification of public health consequences by media exposure. Health Psychology, 39, 355-357. doi:10.1037/hea0000875

Hansen, M., Andersen, T. E., Armour, C., Elklit, A., Palic, S., \& Mackrill, T. (2010). PTSD-8: A short PTSD inventory. Clinical Practice and Epidemiology in Mental Health, 6, 101-108. doi:10.2174/1745017901006010101

Hart, B. L. (1990). Behavioral adaptations to pathogens and parasites: Five strategies. Neuroscience \& Biobehavioral Reviews, 14, 273-294. doi:10.1016/S01497634(05)80038-7

Hofstede, G. (1980). Culture's consequences: International differences in work-related values. . Beverly Hills, CA: Sage. 
Holman, E. A., Thompson, R. R., Garfin, D. R., \& Silver, R. C. (2020). The unfolding COVID19 pandemic: A probability-based, nationally representative study of mental health in the United States. Science Advances, 6, eabd5390. doi:10.1126/sciadv.abd5390

Hopwood, T. L., \& Schutte, N. S. (2017). Psychological outcomes in reaction to media exposure to disasters and large-scale violence: A meta-analysis. Psychology of Violence, 7, 316327. doi:10.1037/vio0000056

Hudson, J. (2006). Institutional trust and subjective well-being across the EU. Kyklos: International Review for Social Sciences, 59, 43-62. doi:https://doi.org/10.1111/j.14676435.2006.00319.x

Huremović, D. (2019). Psychiatry of pandemics: a mental health response to infection outbreak. Cham: Springer International Publishing.

ISTAT. (2021). I.Stat. Retrieved from http://dati.istat.it/Index.aspx?lang=en

Jalloh, M. F., Li, W., Bunnell, R. E., Ethier, K. A., O’Leary, A., Hageman, K. M., . . Redd, J. T. (2018). Impact of Ebola experiences and risk perceptions on mental health in Sierra Leone, July 2015. BMJ Global Health, 3, e000471. doi:10.1136/bmjgh-2017-000471

Kalisch, R., Müller, M. B., \& Tüscher, O. (2015). A conceptual framework for the neurobiological study of resilience. Behavioral and Brain Sciences, 38, e92. doi: 10.1017/S0140525X1400082X

Kessler, R., \& Üstün, T. (2008). The World Health Organization composite international diagnostic interview. In The WHO world mental health surveys: Global perspectives on the epidemiology of mental disorders (pp. 58-90). 
Kye, B., \& Hwang, S.-J. (2020). Social trust in the midst of pandemic crisis: Implications from COVID-19 of South Korea. Research in Social Stratification and Mobility, 68, 100523. doi:https://doi.org/10.1016/j.rssm.2020.100523

LaCour, M. J., \& Vavreck, L. (2014). Improving Media Measurement: Evidence From the Field. Political Communication, 31(3), 408-420. https://doi.org/10.1080/10584609.2014.921258

Lee, S. (2020). Subjective well-being and mental health during the pandemic outbreak: Exploring the role of institutional trust. Research on Aging. Online first. 0164027520975145. doi:10.1177/0164027520975145

Leys, C., Delacre, M., Mora, Y. L., Lakens, D., \& Ley, C. (2019). How to classify, detect, and manage univariate and multivariate outliers, with emphasis on pre-registration. International Review of Social Psychology, 32, 1-10. doi:10.5334/irsp.289

Li, S., Rao, L.-L., Bai, X.-W., Zheng, R., Ren, X.-P., Li, J.-Z., . . Zhang, K. (2010). Progression of the "Psychological Typhoon Eye" and variations since the Wenchuan earthquake. PloS one, 5(3), e9727. doi:10.1371/journal.pone.0009727

Lovibond, P. F., \& Lovibond, S. H. (1995a). The structure of negative emotional states: Comparison of the Depression Anxiety Stress Scales (DASS) with the Beck Depression and Anxiety Inventories. Behaviour Research and Therapy, 33, 335-343. doi:10.1016/0005-7967(94)00075-U

Lovibond, S. H., \& Lovibond, P.F. (1995b). Manual for the Depression Anxiety Stress Scales. (2nd ed.). Psychology Foundation.

Mana, A., \& Sagy, S. (2020). Can political orientation explain mental health in the time of a global pandemic? Voting patterns, personal and national coping resources, and mental 
health during the coronavirus crisis. Journal of Social and Clinical Psychology, 39, 165171. doi:10.1521/jscp.2020.39.3.165

Mancini, A. D. (2020). Heterogeneous mental health consequences of COVID-19: Costs and benefits. Psychological Trauma: Theory, Research, Practice, and Policy, 12(S1), S15S16. doi:10.1037/tra0000894

Mancini, A. D., Aldrich, L., Shevorykin, A., Veith, S., \& John, G. (2020). Threat appraisals, neuroticism, and intrusive memories: A robust mediational approach with replication. Anxiety, Stress, \& Coping, 1-17. doi:10.1080/10615806.2020.1825693

McKinley, J. (2020, March 22). New York City Region Is Now an Epicenter of the Coronavirus Pandemic. New York Times. Retrieved from https://www.nytimes.com/2020/03/22/nyregion/Coronavirus-new-York-epicenter.html

Mehio-Sibai, A., Feinleib, M., Sibai, T. A., \& Armenian, H. K. (2005). A Positive or a Negative Confounding Variable? A Simple Teaching Aid for Clinicians and Students. Annals of Epidemiology, 15(6), 421-423. https://doi.org/https://doi.org/10.1016/j.annepidem.2004.10.004

Mishler, W., \& Rose, R. (2001). What are the origins of political trust?: Testing institutional and cultural theories in post-communist societies. 34(1), 30-62. doi:10.1177/0010414001034001002

Motta Zanin, G., Gentile, E., Parisi, A., \& Spasiano, D. (2020). A preliminary evaluation of the public risk perception related to the COVID-19 health emergency in Italy. International Journal of Environmental Research and Public Health, 17, 3024. doi:10.3390/ijerph17093024 
New York State (2021). New York Forward: Reopening Guidelines. Retrieved from https://forward.ny.gov/new-york-city-phase-four-industries\#malls

O'Laughlin, K. D., Martin, M. J., \& Ferrer, E. (2018). Cross-Sectional Analysis of Longitudinal Mediation Processes. Multivariate Behavioral Research, 53(3), 375-402. https://doi.org/10.1080/00273171.2018.1454822

Prati, G. (2021). Mental health and its psychosocial predictors during national quarantine in Italy against the coronavirus disease 2019 (COVID-19). Anxiety, Stress, \& Coping, 34(2), $145-$ 156. doi:10.1080/10615806.2020.1861253

Prati, G., \& Mancini, A. D. (2021). The psychological impact of COVID-19 pandemic lockdowns: A review and meta-analysis of longitudinal studies and natural experiments. Psychological Medicine, 51, 201-211. doi:10.1017/S0033291721000015

Prati, G., Pietrantoni, L., \& Zani, B. (2011a). Compliance with recommendations for pandemic influenza H1N1 2009: The role of trust and personal beliefs. Health Education Research, 26, 761-769. doi:10.1093/her/cyr035

Prati, G., Pietrantoni, L., \& Zani, B. (2011b). A social-cognitive model of pandemic influenza H1N1 risk perception and recommended behaviors in Italy. Risk Analysis, 31, 645-656. doi:10.1111/j.1539-6924.2010.01529.x

Schmidt, N. B., Martin, A. D., Allan, N. P., Mathes, B. M., Saulnier, K. G., \& March, D. S. (2021). Actual versus perceived infection rates of COVID-19: Impact on distress, behavior and disability. Journal of Psychiatric Research, 137, 525-533. doi:10.1016/j.jpsychires.2021.03.061

Sibley, C. G., Greaves, L. M., Satherley, N., Wilson, M. S., Overall, N. C., Lee, C. H. J., . . . Barlow, F. K. (2020). Effects of the COVID-19 pandemic and nationwide lockdown on 
trust, attitudes toward government, and well-being. American Psychologist, 75, 618-630. doi:10.1037/amp0000662

Strömbäck, J., Djerf-Pierre, M., \& Shehata, A. (2016). A question of time? A longitudinal analysis of the relationship between news media consumption and political trust. International Journal of Press/Politics, 21, 88-110. doi:10.1177/1940161215613059

Thompson, R. R., Garfin, D. R., Holman, E. A., \& Silver, R. C. (2017). Distress, worry, and functioning following a global health crisis: A national study of Americans' responses to Ebola. 5(3), 513-521. doi:10.1177/2167702617692030

Thomson Jr., R. A., Bolger, D., \& Ecklund, E. H. (2019). A tale of two populisms: Divergent and convergent correlates of trust in Congress and corporations. Conference Papers -American Sociological Association, 1-34.

Triandis, H. C. (1995). Individualism and collectivism. Boulder, CO: Westview Press.

Triandis, H. C., \& Gelfand, M. J. (1998). Converging measurement of horizontal and vertical individualism and collectivism. Journal of Personality and Social Psychology, 74(1), 118-128.

United States Census Bureau (2019). Quick facts: New York City, New York. Retrieved from https://www.census.gov/quickfacts/newyorkcitynewyork

Winfield, N. (2020). A pandemic atlas: How Italy becomes Europe's viral epicenter. Associated Press. Retrieved from https://apnews.com/article/pandemics-europe-italy-healthcoronavirus-pandemic-f71955b0327aa9f7a0c2e44b9f485475

Wright, D. B., London, K., \& Field, A. P. (2011). Using bootstrap estimation and the plug-in principle for clinical psychology data. Journal of Experimental Psychopathology, 2, 252270. doi:10.5127/jep.013611 
Xie, X. F., Stone, E., Zheng, R., \& Zhang, R. G. (2011). The 'Typhoon Eye Effect': determinants of distress during the SARS epidemic. Journal of Risk Research, 14(9), 1091-1107. doi: $10.1080 / 13669877.2011 .571790$

Yang, Y., Tang, M., Zhou, W., \& Huhe, N. (2014). The Effect of Media Use on Institutional Trust in China. Problems of Post-Communism, 61, 45-56. doi:10.2753/PPC10758216610304

Zhao, H., He, X., Fan, G., Li, L., Huang, Q., Qiu, Q., . . Xu, H. (2020). COVID-19 infection outbreak increases anxiety level of general public in China: involved mechanisms and influencing factors. Journal of Affective Disorders, 276, 446-452. doi:10.1016/j.jad.2020.07.085 


\section{Footnotes}

${ }^{1}$ Note that our preregistered analysis stipulated COVID-19 worry as a mediator of the effect of prevalence region on mental health outcomes. We tested these effects as preregistered. However, because we now view mediational analysis as fundamentally misleading in a cross-sectional design (O'Laughlin et al., 2018), exploratory analyses treated COVID-19 worry as an explanatory variable rather than a mediator. See data analysis section for more detail. ${ }^{2}$ The findings were almost entirely unchanged when comparing the five-item and six-item scale. One exception was the emergence of a significant interaction effect of Region x COVID-19 worry in predicting PTSD symptoms in the United States $(p=.04)$. With the six-item measure, this finding was only marginal $(p=.09)$. Given that the five-item measure had superior reliability, this difference is likely attributable to reduced measurement error in the five-item version of the scale.

${ }^{3}$ We also observed significant variation in individualism across prevalence regions, $F(3,866)=$ $10.30, p<.001$. Pairwise comparisons showed that New York had higher individualism than every other region $\left(p_{\mathrm{s}}>\right.$.006), suggesting that country-level differences were attributable to high individualism in New York.

${ }^{4}$ We interpreted these effects as evidence of confounding bias, because media exposure accounted for the effects of institutional trust. By contrast, we did not interpret media exposure as a confound in the Country $\mathrm{x}$ Region interaction predicting COVID-19 worry. Instead, because both country and region must be represented by other factors, media exposure appeared to serve as an explanatory variable for this particular interaction effect. 


\section{Table 1}

Comparisons of relevant demographic and study variables in high (New York City; Lombardy) and low prevalence (San Francisco; Campania) regions in the United States and Italy

\begin{tabular}{|c|c|c|c|c|c|c|}
\hline \multirow[b]{2}{*}{ Variable } & \multicolumn{2}{|c|}{ United States } & \multicolumn{2}{|c|}{ Italy } & \multirow[b]{2}{*}{ NY vs. SF } & \multirow[b]{2}{*}{$\begin{array}{l}\text { Lombardy vs. } \\
\text { Campania. }\end{array}$} \\
\hline & $\begin{array}{l}\text { New York City } \\
\quad(n=210)\end{array}$ & $\begin{array}{c}\text { San } \\
\text { Francisco } \\
(n=205) \\
\end{array}$ & $\begin{array}{l}\text { Lombardy } \\
(n=231)\end{array}$ & $\begin{array}{l}\text { Campania } \\
(n=224)\end{array}$ & & \\
\hline Age ${ }_{\text {ac }}$ & $44.82(17.20)$ & $44.32(17.03)$ & $45.55(14.41)$ & $44.82(14.21)$ & $U=21167.00$ & $t=.55$ \\
\hline Female gender $\mathrm{b}$ & $50.7 \%$ & $52.4 \%$ & $50 \%$ & $50 \%$ & $\chi^{2}=.44$ & $\chi^{2}=.05$ \\
\hline Education & $3.41(1.03)$ & $3.46(1.19)$ & $2.18(1.35)$ & $2.13(1.32)$ & $U=21031.00$ & $U=25340.50$ \\
\hline Institutional trust & $4.39(1.70)$ & $4.20(1.76)$ & 4.39 (1.47) & $3.72(1.09)$ & $t=1.12$ & $t=5.49 * * *$ \\
\hline General distress & 20.47 (18.68) & $16.30(15.11)$ & $17.90(11.52)$ & $20.60(13.43)$ & $t=3.65^{* * *}$ & $t=-3.55^{* * *}$ \\
\hline Individualism & $5.81(1.18)$ & $5.35(1.18)$ & $5.32(1.09)$ & $5.50(1.04)$ & $t=3.87 * * *$ & $t=1.75$ \\
\hline $\begin{array}{l}\text { Political } \\
\text { identification }{ }_{\mathrm{d}}\end{array}$ & $3.79(1.81)$ & 3.34 (1.69) & $4.30(1.68)$ & 3.68 (1.64) & $t=3.79^{* *}$ & $t=4.29 * *$ \\
\hline
\end{tabular}

Note: NY = New York City; SF = San Francisco. a, Age was measured categorically in the United States (mean and standard deviation based on imputed midpoint of each category) bThree participants reported their gender as "other." c Age, income and education were compared using Mann-Whitney U tests. d Lower scores indicate more liberal beliefs. For ease of comparison, we report the categorical means for income and education. See Supplemental Table S1 for full descriptive comparison data. $* * * p<.001 ; * * p<.01$ 


\section{Table 2}

Effects of Prevalence Region, Country, and their Interaction on General Distress, PTSD

Symptoms, and COVID-19 Worry (Bootstrapped General Linear Models)

\begin{tabular}{lllll}
\hline BCa $95 \% \mathrm{CI}^{\mathrm{a}}$ & $p$ & $\eta^{2}$ \\
\hline
\end{tabular}

General distress

$\begin{array}{llccr}\text { Country }^{\mathrm{b}} & 2.45 & {[-.73,5.70]} & .123 & .00 \\ \text { Prevalence region }^{\mathrm{c}} & 2.53 & {[0.35,5.03]} & .046 & .00 \\ \text { Country } \times \text { prevalence region } & -6.65 & {[-11.18,-2.70]} & .006 & .02\end{array}$

PTSD

$\begin{array}{lcccc}\text { Country }^{\mathrm{b}} & 0.45 & {[0.31,0.59]} & <.001 & .05 \\ \text { Prevalence region }^{\mathrm{c}} & 0.22 & {[0.10,0.26]} & <.001 & .01 \\ \text { Country } \times \text { prevalence region } & -0.51 & {[-0.68,-0.35]} & .002 & .03\end{array}$

COVID-19 worry

$\begin{array}{lcccc}\text { Country }^{\mathrm{b}} & 0.46 & {[0.23,0.69]} & <.001 & .02 \\ \text { Prevalence region }^{\mathrm{c}} & 0.23 & {[0.08,0.48]} & .003 & .01 \\ \text { Country } \times \text { prevalence region } & -0.68 & {[-1.01,-0.34]} & <.001 & .02\end{array}$

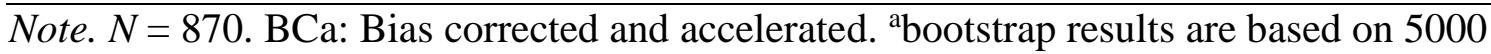
bootstrapped samples. ${ }^{\mathrm{b}}$ country was coded 0 for USA and 1 for Italy. ${ }^{\mathrm{c}}$ prevalence region was coded 0 for regions with low risk and 1 for regions with high risk. Per our preregistered plan, all analyses controlled for income. The inclusion of additional demographic covariates did not alter the interpretation of the results. 


\section{Figure 1}

Cumulative deaths from COVID-19 (per 100,000 population) and timing of data collection in the United States (top panel) and Italy (bottom panel).
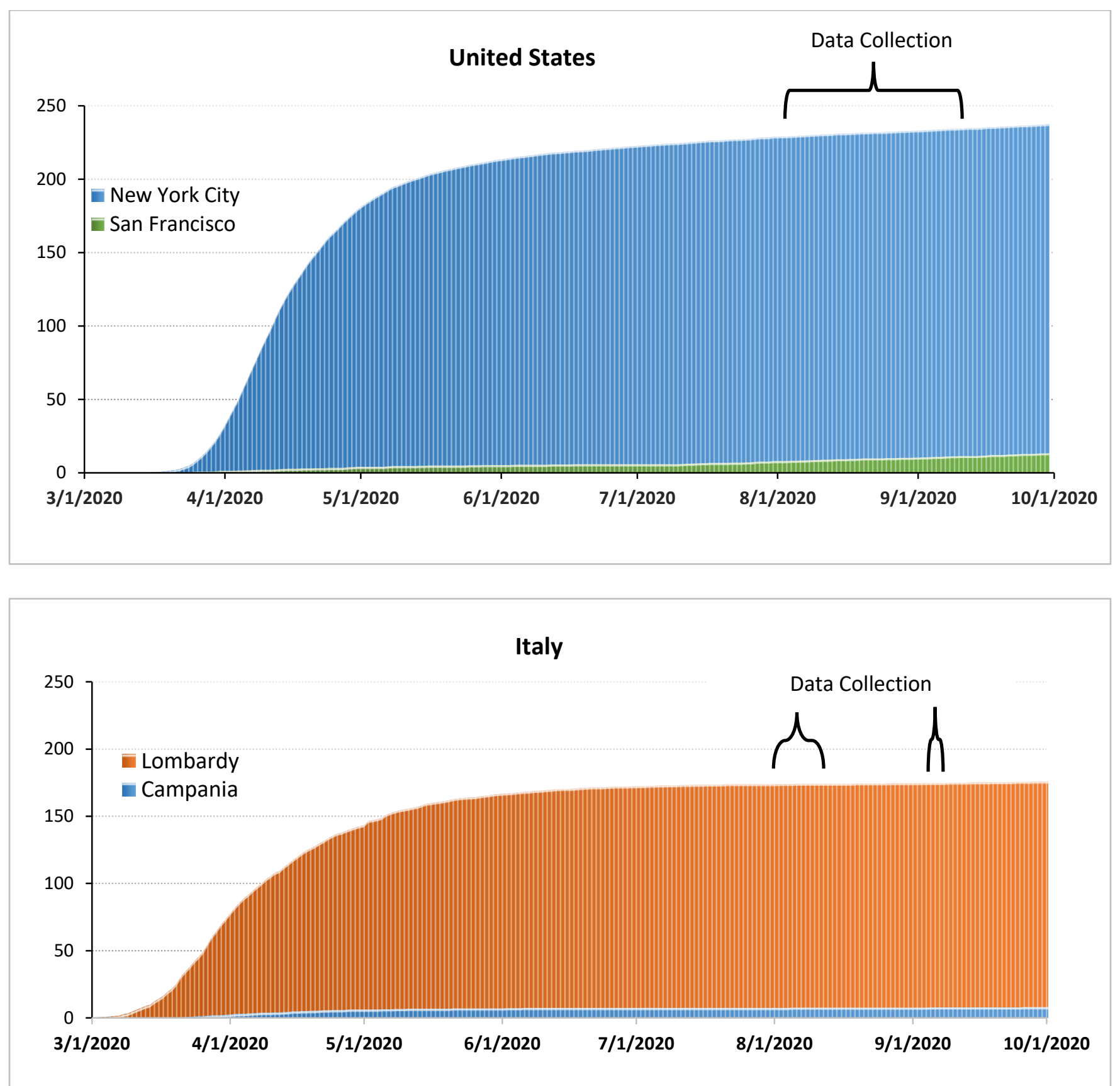

Note: New York City data were collected from July 29th to August 24th ( $M d=$ August 9th; $M=$ August 7 th, $S D=6.18$ days). San Francisco data were collected from July 29th to September 10th (Md= August 19th; $M=$ August 18th, $S D=9.10$ days). Lombardy and Campania data were collected from July 31 st to August 4th, and a small proportion of data (4.4\%) across regions was collected in early September $(M d=$ July 31 st, $M=$ August 2 nd, $S D=6.18$ days). 


\section{Figure 2}

PTSD (top panel), general distress (middle panel), and COVID-19 worry (bottom panel) by COVID-19 region (low and high prevalence) and country.
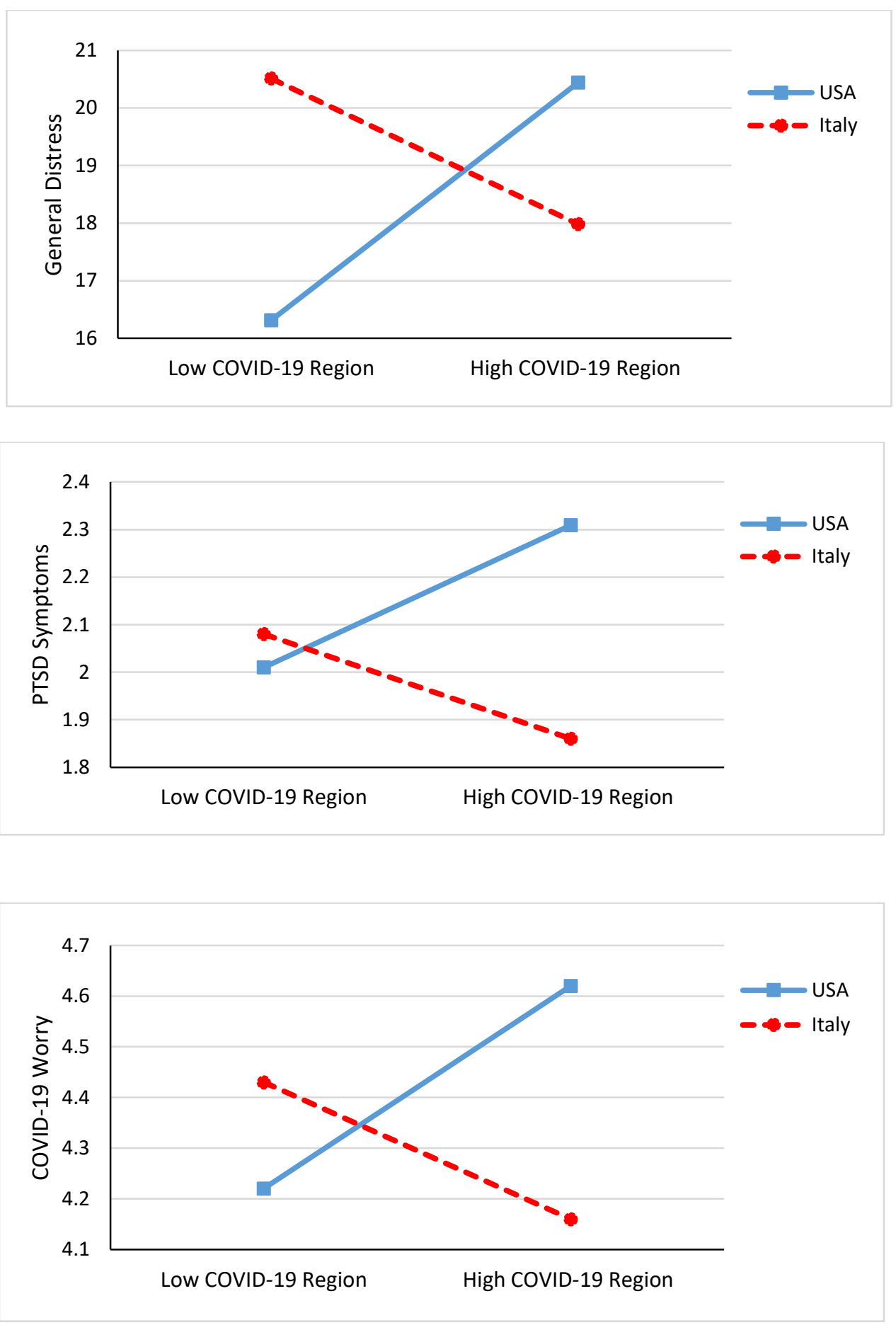


\section{Figure 3.}

General distress (top panel) and PTSD (middle panel) in United States and PTSD symptoms in Italy (bottom panel) by COVID-19 prevalence region and COVID-19 worry (low = $-1 S D$; high $=+1 S D)$.
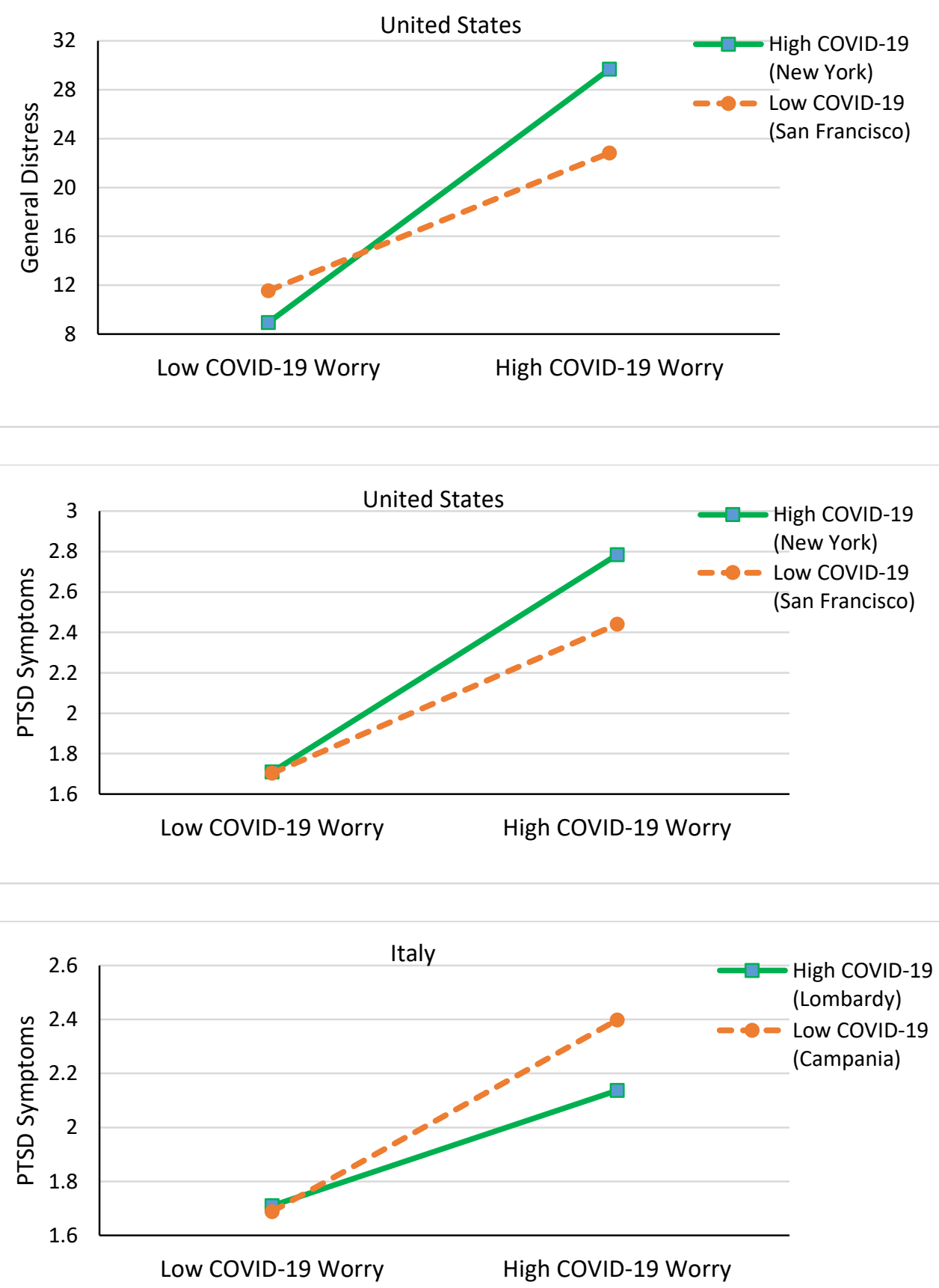